\title{
AIR QUALITY PREDICTION using SUPERVISED MACHINE LEARNING TECHNIQUE
}

\author{
1.K.Ramamohanarao,2.D.Joshna, 3.K.Madhurya, 4.K.Srividya 1.Assistant \\ Professor, 2,3,4-Students 1.it.mohan@sasi.ac.in 2.jyoshna1212@sasi.ac.in 3. \\ madhurya1228@sasi.ac.in 4. srividhya1218@sasi.ac.in, Sasi Institute of \\ Technology \& Engineering, Tadepalligudem
}

\begin{abstract}
:
Generally, air contamination alludes to the arrival of different contamination into the air which are compromising the human wellbeing and planet also. The air contamination is the major hazardous horrendous to humankind at any point confronted. It causes major harm to creatures, plants and so forth, if this 'continues proceeding, the individuals will confront major circumstances in the forthcoming years. The significant toxins are from the vehicle and enterprises. In this way, to forestall this issue significant areas need to foresee the air quality from transport and ventures .In existing undertaking there are numerous hindrances. The venture is tied in with assessing the PM2.5 fixation by planning a photo based strategy. In any case photographic technique isn't the only one adequate to compute PM2.5 since it contains just one of the grouping of toxins furthermore, it ascertains just PM2.5 so there are some passing up a great opportunity of the significant toxins and the data required for controlling the contamination .So along these lines we proposed the Al procedures by UI of GUI application. In this numerous dataset can be joined from the diverse source to shape a summed up dataset and different Al calculations are used to get the outcomes
\end{abstract}

with the most extreme precision. From looking at different Al calculations we can get the best precision result. Our assessment gives the thorough manual to affectability assessment of model boundaries concerning generally speaking execution in forecast of air great contaminations through exactness computation. Furthermore to examine and think about the presentation of $\mathrm{Al}$ calculations from the dataset with assessment of GUI based UI air quality forecast by credits.

Keywords : Toxins dataset, GUI results, AI, directed calculation.

\section{INTRODUCTION :}

Machine Learning(ML) technique is utilized to foresee what's to come from the past information. $\mathrm{ML}$ is a segment or sort of $\mathrm{Al}$ that gives PCs the capacity to learn without being customized. ML procedure is the one that can change when presented to new information. ML is only the capacity to learn by itself and shows the PC how to react to a contribution by itself. It contains numerous calculations into it .The calculations are grouped into 3 primary classifications : supervised learning, unsupervised learning and reinforcement learning. Supervised learning is the undertaking of learning capacity that makes a contribution to a yield dependent on illustration of input pairs and 
output pairs. The fundamental component of supervised calculation is becoming acquainted with a set of rules, investigating the preparation records and creating a deduced description which can be utilized for planning new models. Unsupervised learning is utilized to draw inductions from dataset composed of info information without named reactions. Its fundamental technique is group examination which can be utilized for exploratory information investigation to discover stowed away examples or gathering in information .Finally, support learning worries about how programming specialists should make a move in a climate to augment some thought

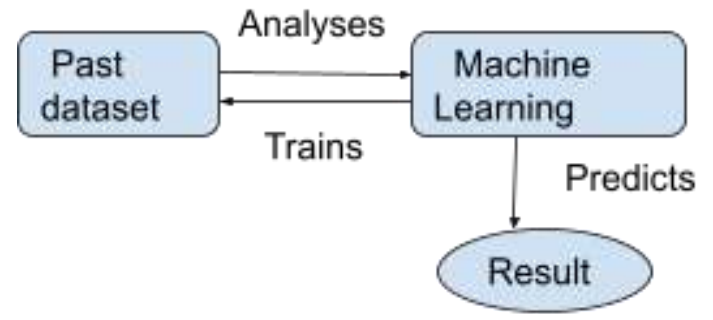

\section{PROCESS OF MACHINE LEARNING}

of aggregate prize.

To assess the PM2.5 focus by planning a photo based strategy. By perception it is tracked down that the nature of air in immersion maps crediting totally extraordinary approaches on high and low fixations. They watch out for misfortune their substances and for the most part the pixel esteems will in general be zero under high fixation. At the point when they attempt to make it comparative the nature of primary data misfortunes. The utilization of weibull conveyance can determine the worth to appraise the shading data. The photographic strategy isn't adequate to compute PM2.5 fixation [1] [9]. It takes just one worth of the poisons noticeable all around. It doesn't figure out all the poisons. Air poisons are a significant danger to human culture. They will be major for making illness human too to the living beings on earth. On the off chance that this circumstance must be defeated the contamination causing poisons ought to be discovered. [6][4] To conquer this impediment from the existing framework we have carried out the method of ML. [5] Different calculations from the $\mathrm{ML}$ are utilized for getting the best outcome. The ML approach by client interface of GUI application is utilized as a proposed framework. They are utilized to examine the various dataset from the various sources and all are consolidated to frame the summed up dataset. The cycle from the start characterizes an issue of what the individuals are confronting and setting up the dataset from the past report and afterward assessing the dataset like eliminating invalid values, rehashed qualities, greatest and least toxins and so on, then, at that point frequently acquiring the assessment of calculation will happens. By contrasting the equivalent dataset and different $\mathrm{ML}$ calculations it tracks down the best outcome from the examination and predicts the outcome for the analyzer by GUI .

\section{LITERATURE REVIEW}

GuanghuiYue ,KeGu, and JunfeiQiao, Member, have suggested that PM2.5 focus by planning a photo based strategy. It is tracked down that the immersion map is delicate to air quality, displaying all together various appearances under high and low PM2.5 focuses. To process the inclination likeness between the immersion and dim scale guides to measure the primary data misfortune. Using the Weibull circulation to fit the immersion guide and ready to infer a worth to assess the shading data. At last, the PM2.5 convergence of a picture can be assessed through the mix of the previously 
mentioned two highlights followed by a nonlinear planning strategy.Both mathematical and envisioned outcomes on genuine caught information approve the adequacy and prevalence of the proposed technique in correlation with the important state-of-the-craftsmanship techniques. Air contamination has become an around the world concerned issue and natural assessment of air quality can give a positive direction to both individual and modern practices. [1] Ishan Verma, Rahul Ahuja and Hardik Meisheri, proposed the technique which state about idea of Recurrent Neural Networks (RNN) has end up being exceptionally effective in handling fleeting information It is hard to acquire ideal converging since various organizations prepared on a similar information can at this point don't be viewed as autonomous it proposed bidirectional repetitive neural organization (BRNN) that can be prepared utilizing all accessible info data before and eventual fate of a particular time period. [2] Temesgen Walelign Ayele, Rutvik Mehta, proposed that these days it is better if each activity is finished utilizing new innovation to fulfill the interest of person, Association, Enterprise and so on Web of Things (loT) is one of the primary correspondence improvements somewhat recently. Through this idea, it is feasible to interface endless low-fueled keen inserted objects to one another and to the Web. [3] Luke Curtis, William Rea, Patricia Smith-Willis, suggested that the objective of this survey is to briefly sum up a wide scope of the new exploration on wellbeing impacts of numerous sorts of outside air contamination. An assessment of the wellbeing outcomes of primary outside air contamination which incorporates particulates, carbon monoxide, sulfur and nitrogen oxides, corrosive gases, metals, unstable organics, solvents, pesticides, radiation and bio vaporizers is introduced. [4] Khaled Bashir Shaban, Abdullah Kadri and Eman Rezk, recommended that air quality information be gathered remotely from observing bits that are furnished with a variety of vaporous meteorological sensors. These information are examined and utilized in determining fixation upsides of contaminations utilizing astute machine to machine stage. The stage employs MLbased calculations to fabricate the gauging models by gaining from the gathered information. [5]

\section{III.PROPOSED METHODOLOGY}

Checking and maintaining air palatable has found itself to be perhaps the foremost essential exercises in numerous modern and substantial regions today. The exception of air is antagonistically influenced in light of various assortments of contamination due to transportation, power, fuel utilization and so forth. The statement of destructive gases is making a real danger for the personal satisfaction in savvy urban communities. With expanding air pollution, we need to execute productive air quality checking models which gather data about the convergence of air contaminations and provide evaluation of air contamination in each region. this framework with Concept of Recurrent Neural Organizations (RNN) [2] has find yourself being extremely effective in preparing worldly information it's hard to induce ideal converging since various organizations prepared on an identical information can presently do not be viewed as autonomous For this they're utilizing the sensor which are required observed consistently that they're working don't seem to be. [3][8] For this sort of issue utilization of equipment is kept removed from in the proposed model. One among the present framework offers the forecast of air quality 
following 1 hour [7], they deferral of such a strategy are tried to not by a boa pilot which gives the instant result. Presently the observation of air and safeguarding air quality has become the foremost fundamental movement on numerous spaces like metropolitan and mechanical regions. The character of air has become unfavorably influenced due to the various sorts of contamination from transportation, ventures, coal products[10], the statement of destructive gases on to the air makes the real danger to human existence with the expanding air contamination we want to find the arrangement. By gathering the information about the poisons what's more, give the last report back to every region about the current state of their space. Therecorda are submitted to Indian meteorological areas utilizing Al strategies. The expectations are from air quality list esteem. The dataset about the air toxins are taken as an information and afterward going into the cycle of data preparing.

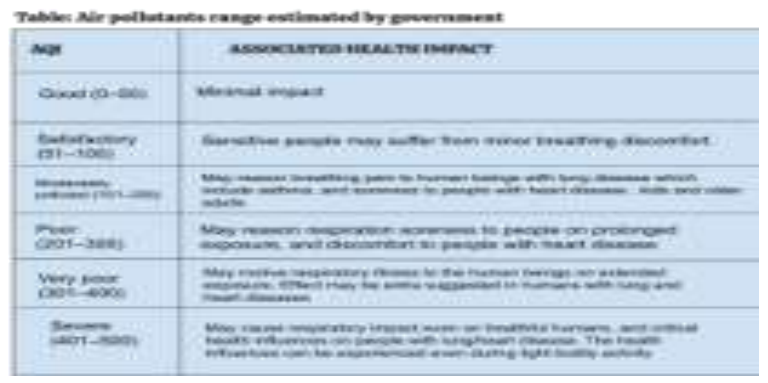

IV.BLOCK DIAGRAM

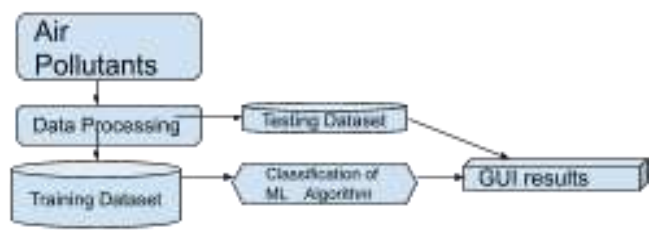

BLOCK DIAGRAM OF PROPOSED METHODOLOGY

The dataset which are going into the information preparing stage are going through the way toward discovering the information shape, information type, end of invalid qualities and so forth, the yield from the information preparing is acquired as an appropriate dataset with right values and no rehashed values. Then, at that point these are going into the preparation and testing of the dataset.

Here the dataset is prepared to the machine and tried by machine. Then, at that point the prepared dataset goes into the machine learning calculations where numerous calculations are thought about for tracking down the best precision result. The managed Machine learning calculations are utilized and the last yield is shown in the GUI interface.

V.

PHASE

A. Data approval and pre-processing strategy

B. Investigation data analysis of perception and training a model by given ascribes

C. Execution estimations of Logistic regression and Naive Bayes algorithm

D. Execution estimations of Random Forest and Support Vector Machines

E. Result in GUI

\section{A. Data Approval and pre-processing strategy :}

This interaction used to get the mistake pace of the machine learning model can be considered as near the genuine mistake pace of the dataset. In the event that the information volume is adequately enormous to the populace, it isn't required for approval procedure. Yet, in reality, working with the example of information that might not have a genuine delegate of the given dataset. Tracking down the missing qualities, copied qualities and insight regarding information type for example regardless of whether buoy or number and so on Information assortment, information investigation and the way toward tending to information 
content quality and construction can be amount to a tedious measure. The information cleaning measure is finished by utilizing python's pandas library. They explicitly center around the greatest information cleaning task, missing worth and it is all the more fast clean information process.it sets aside less effort for cleaning. Separating the library bundles with a given dataset. The examinations are finished by their information shape, information type, assessing missing qualities and copy values.

Variable recognizable proof with Univariation, Bi-variation and Multi-variation investigation: It used to track down the missing upsides of information outline, discovering copy esteems, discovering remarkable qualities, discover tally upsides of information outline. It clarifies the information outline, given the dataset. It disposes of the additional segments and rename and drop the given information frame.it determines the kind of values.

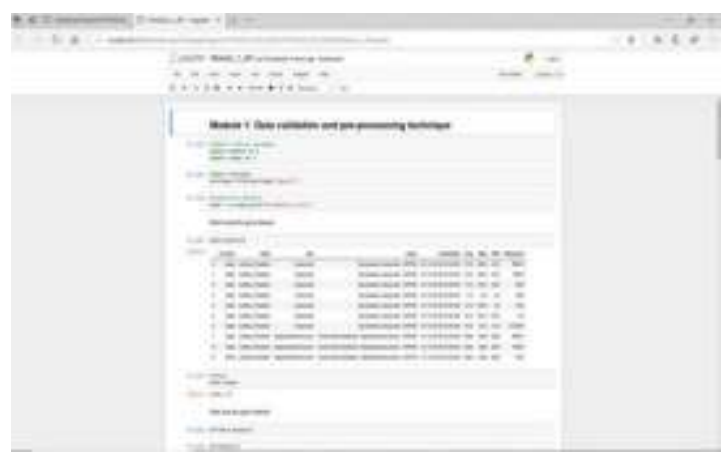

Importing Python Packages

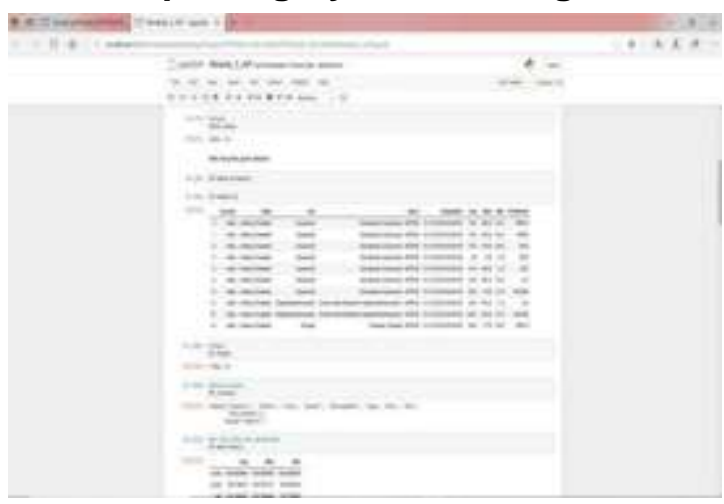

\section{Omitting null and recursive values}

B. Investigation data analysis of perception and training a model by given ascribes :

Data representation is a vital expertise in applied measurements and gadget learning. Information representation gives a significant set-up of apparatuses for acquiring a subjective understanding. This can be useful while investigating and becoming more acquainted with a dataset and can assist with distinguishing designs, degenerate information, anomalies, and substantially more.

1. Instructions to diagram time series information with line plots and downright amounts with bar diagrams.

2. Instructions to sum up data dispersions with histograms and box plots.

3. Step by step instructions to sum up the connection between factors with dissipate

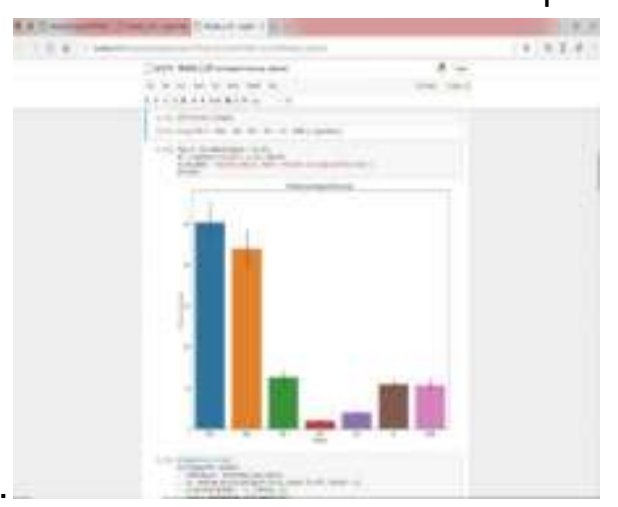

plots.

Fig: estimating the dataset values in bar chart

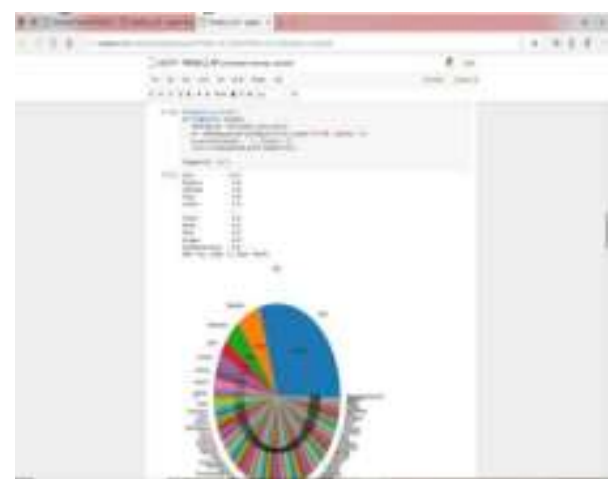

Fig: Estimating the country's dataset values in pie chart 


\section{Execution estimations of Logistic} regression and Naive Bayes algorithm : Computation of exactness from various calculation

\section{Algorithm}

\subsection{Logistic regression :}

It is a measurable technique for investigating a measurement set in which there is at least one impartial factor that decides an eventual outcome. The endproduct. The eventual outcome is estimated with a dichotomous variable (where there are best two plausible results). The motivation behind calculated relapse is to track down the palatable fitting adaptation to clarify the relationship among the dichotomous element of interest (based variable $=$ response or result variable) and a fixed of unbiased (indicator or informative) factors. Calculated relapse is an ML characterization calculation that is utilized to expect the chance of an absolute organized variable.

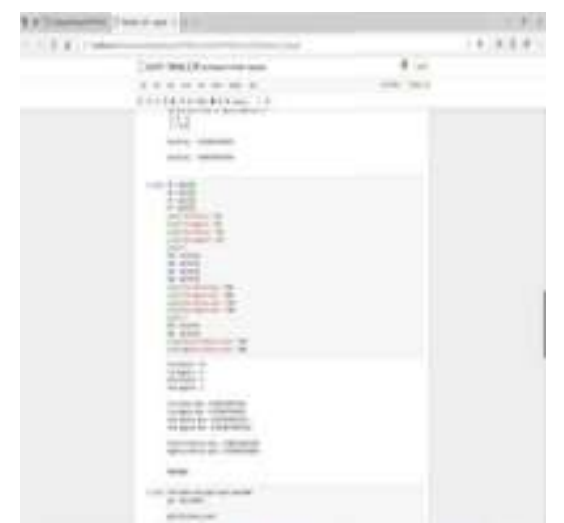

Fig : calculation of values by logistic regression algorithm and finding the accuracy value through algorithm

\subsection{Naive Bayes:}

Naive Bayes model is easy to construct and especially valuable for extremely enormous insights sets. Alongside effortlessness, its miles are perceived to beat even exceptionally refined arrangement techniques. It is straightforward and quick to expect the style of the test data set. It likewise performs pleasantly in multi class forecasts when suspicion of freedom holds, a Naïve Bayes classifier performs better access to different models like strategic relapse and needs less preparing records. It performs property in the event of downright input factors contrasted with mathematical variables, ordinary dispersion is accepted (chime bend, that is a strong presumption).

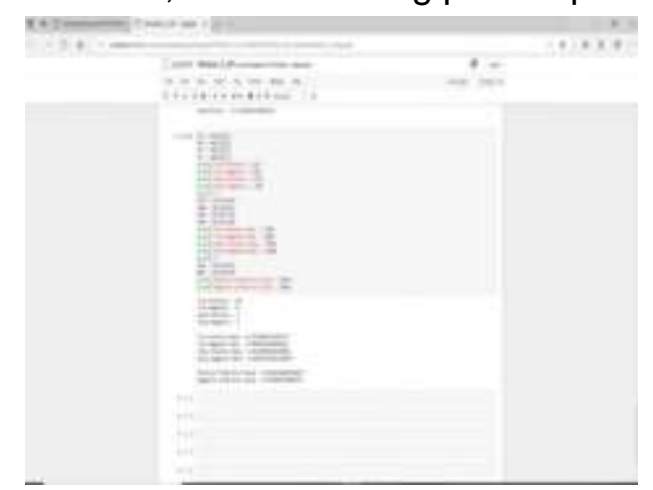

Fig: Calculation of accuracy values through naive Bayes algorithm

D. Execution estimations of Random Forest and Support Vector Machines

2.3 Random Forest :

Random Forest is a kind of supervised framework considering calculation principally dependent on group acquiring information on troupe. Learning is a kind of acquiring information in which you are essential for various kinds of calculation or equivalent calculation of different occurrences to shape a more powerful expectation model. The irregular woods region set of rules might be utilized for every relapse and class errands.

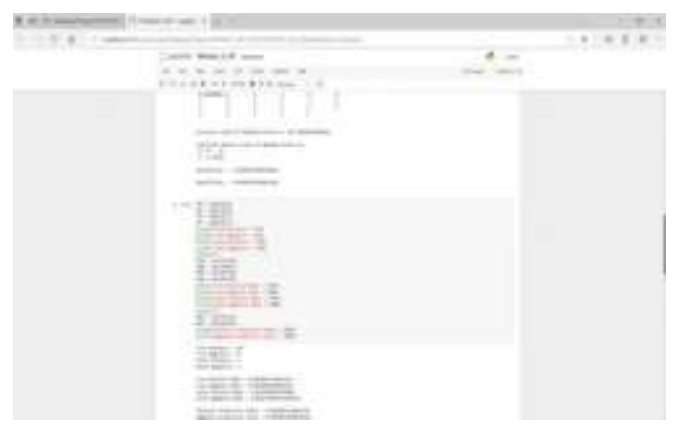




\section{Fig: Calculation of accuracy values} through Random forest algorithm

The accuracy values are calculated for all algorithm though the general formula : Accuracy $=(T P+T N) /(T P+T N+F P+$ FN)

Precision $=$ TP $/(T P+F P)$

True Positive Rate $(T P R)=T P /(T P+F N)$ False Positive $(F P R)=$ FP $/(F P+T N)$ TP True Positive FP - False Positive

\section{VI.FLOWCHART}

Here is the flowchart determines the process of prediction

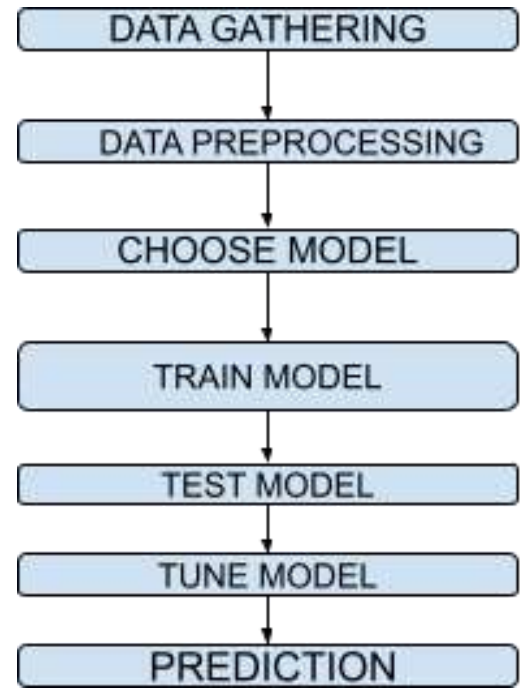

Flowchart of the prediction process

\section{E.Result in GUI :}

The last yield contains the module state, city, air quality record esteem by client and contamination forecast esteem, hotspot for contamination and AQI stages are recorded.

\section{CONCLUSION}

The scientific interaction started from records cleaning furthermore, preparing,missing worth, exploratory investigation and hence model construction and assessment. The wonderful exactness on open take a see set is better precision rating can be found out. This application can help India meteorological branch in anticipating the future of air best and its notoriety and relies upon that they can make a move.

\section{REFERENCES}

1. Guanghui Yue, Ke Gu, and Junfei Qiao, Member," Effective and Efficient Photo -Based PM2.5 Concentration Estimation", website:

http://www.ieee.org/publications_stand ards/publications/rights/inde $\mathrm{x} . \mathrm{html}$

2. Ishan Verma, Rahul Ahuja and Hardik Meisheri, "Air pollutant severity prediction using Bi-directional LSTM Network" website: https://ieeexplore.ieee.org/abstract/doc ument/8609664

3. TemeseganWalelign Ayele, Rutvik Mehta, "Air pollution monitoring and prediction using loT" website:

https://ieeexplore.ieee.org/abstract/doc ument/8473272

4. Luke Curtis, William Rea, Patricia Smith-Willis, "Adverse health effects of outdoor air pollutants", website : https://www.sciencedirect.com/science /article/pii/S0160412006000444

5. Khaled Bashir Shaban, Abdullah Kadri and Eman Rezk," Urban Air Pollution Monitoring System with Forecasting Models", website: https://ieeexplore.ieee.org/abstract/doc ument $/ 7370876$

6. C. A. Pope, III, et al., "Lung cancer, cardiopulmonary mortality, and long-term exposure to fine particulate air pollution," J. Amer. Med. Assoc., vol. 287, no. 9, pp. 1132-1141, 2002.

7. K. Gu, J. Qiao, and W. Lin, "Recurrent air quality predictor based on meteorology- and pollution-related factors," IEEE Trans. Ind. Informat., vol. 14, no. 9, pp. 3946-3955, Sep. 2018.

8. G. Andria, G. Cavone, V. Di Lecce, and A. M. L. Lanzolla, "Model characterization in measurements of environmental pollutants via data correlation of sensor outputs," IEEE 
Trans. Instrum. Meas., vol. 54, no. 3, pp. 1061-1066, Jun. 2005.

9. K. He, J. Sun, and X. Tang, "Single image haze removal using dark channel prior," IEEE Trans. Pattern Anal. Mach. Intell., vol. 33, no. 12, pp. 2341-2353, Dec. 2011. 10. Y. Zhao, S. Wang, L. Duan, Y. Lei, P. Cao, and J. Hao, "Primary air pollutant emissions of coal-fired power plants in China: Current status and future prediction," Atmos. Environ., vol. 42, no. 36, pp. 8442-8452, 2008. 